\title{
On quadratic bond-order decomposition within molecular orbital space
}

\author{
Dariusz Szczepanik • Janusz Mrozek
}

Received: 22 June 2012 / Accepted: 18 March 2013 / Published online: 29 March 2013

(C) The Author(s) 2013. This article is published with open access at Springerlink.com

\begin{abstract}
A simple method of analysing and localization of canonical molecular orbitals for particular chemical bond using the MO-resolved bond-order decomposition scheme is presented. An alternative definition of classical bond order orbitals is provided and links to communication theory of the chemical bond are outlined and briefly discussed. The introduced procedure of decomposition of quadratic bond orders allows one to analyse two- as well as three- center chemical bonds within the framework of the same theory.
\end{abstract}

Keywords Wiberg's index - Bond order decomposition - Localized orbital . Molecular orbital $\cdot$ Scattering operator $\cdot$ Communication theory

\section{Introduction}

The concept of chemical bond order [1] is deeply embedded in chemical intuition. Bond multiplicities are widely used in evaluating of electronic structure of molecules despite the fact, that they are not observables in quantum-mechanical sense. The main reason of their popularity is that they provide a very compact tool for rationalizing the results of theoretical calculations.

According to the qualitative molecular orbital (MO) theory [2,3] the formal bond order (multiplicity) in diatomics is given by

$$
B_{X Y}=\frac{1}{2}\left(N-N^{*}\right),
$$

D. Szczepanik $(\varangle) \cdot$ J. Mrozek

Department of Computational Methods in Chemistry, Jagiellonian University,

R. Ingardena St. 3, 30-060 Cracow, Poland

e-mail: szczepad@chemia.uj.edu.pl 
where $N$ and $N^{*}$ stand for the numbers of electrons occupying bonding and antibonding molecular orbitals, respectively. This equation can be also rewritten in the following form:

$$
B_{X Y}=\sum_{i}^{M O} B_{X Y}^{(i)}=\frac{1}{2} \sum_{i}^{M O} n_{i} \Theta_{X Y}^{(i)}
$$

where $n_{i}$ is a number of electrons occupying molecular orbital $\psi_{i}$ and $\Theta_{X Y}^{(i)}$ denotes a contribution of this particular orbital to the bond order $B_{X Y}$. In the case of simple qualitative models of diatomic species the quantity $\Theta_{X Y}^{(i)}$ holds one of the three admissible values: $\Theta_{X Y}^{(i)}=0$ (non-bonding MO), 1 (bonding MO) and -1 (anti-bonding $\mathrm{MO})$.

However, in typical ab initio calculations canonical MOs of poliatomics are delocalized over the whole molecule and thus one MO can simultanously reveal bonding character with respect to particular chemical bond and anti-bonding character with respect to another. There is a variety of computationaly efficient methods of localization and decomposition of the chemical bonds into $\sigma, \pi$ and $\delta$ components: tranformation $\mathrm{MO} \rightarrow$ LMO by optimalization the expectation value of an appropriate operator [4-6], transformation $\mathrm{MO} \rightarrow \mathrm{NBO}$ into the natural bond orbital (NBO) representation [7], transformation $\mathrm{MO} \rightarrow \mathrm{LOBO}$ into the localized orbitals of bond order (LOBO) [8], symmetry based bond-order decomposition techniques [9], etc. These methods allow one to gain a very compact and intuitive picture of typical chemical bonds but sometimets (especially in the case of three-center two-electron chemical bonds, delocalized bond, very weak atomic interactions, etc.) they can lead to unreasonable results.

In this paper we introduce a simple method of analysing and localization of canonical molecular orbitals (CMO) for particular chemical bonds using MO-resolved bond order decomposition scheme and the bond order orbitals (BOO) concept [10]. Also, the relation to the communication theory of the chemical bond (CTCB) [11-20] is outlined and briefly discussed.

\section{MO-resolved decomposition}

The most popular and commonly used in quantum-chemical calculations are quadratic bond orders and atomic charges based on the reduced density matrix $\boldsymbol{\gamma}$ (also known as charge and bond-order matrix, CBO matrix [21]) which constitutes the key quantity of the standard one-determinantal description in the atomic orbital representation. For closed-shell systems, using Löwdin's symmetrically orthogonalized atomic orbitals (AO,OAO) [22], $\chi=\left\{\chi_{k}\right\}$, matrix $\boldsymbol{\gamma}$ reads:

$$
\boldsymbol{\gamma}=\mathbf{C n C}^{\dagger}=\left\{\gamma_{k, l}=2\left\langle\chi_{k}\left|\hat{P}_{\psi}^{o}\right| \chi_{l}\right\rangle=2 \sum_{i=1}^{o c c} C_{k, i} C_{l, i}\right\},
$$


where $\mathbf{C}$ stands for the LCAO MO coefficients matrix [23,24], $\mathbf{n}=\left\{n_{i}=0,2\right\}$ is a diagonal matrix collecting electron occupation numbers of each molecular orbital $\psi$ and thus determines the reference electron configuration. In Eq. (3) $\hat{P}_{\psi}^{o}$ represents the projection operator onto the subspace of all occupied molecular orbitals, $\psi^{o}=\left\{\psi_{i}^{o}\right\}$ :

$$
\hat{P}_{\psi}^{o}=\sum_{i=1}\left|\psi_{i}^{o}\right\rangle\left\langle\psi_{i}^{o}\right|=\sum_{i=1}^{o c c} \hat{P}_{i}
$$

In other words, the element $\gamma_{k, l}$ of the CBO matrix is determined by the projection of state $\left|\chi_{l}\right\rangle$ onto the state $\left|\chi_{k}\right\rangle$ through the subspace of (doubly) occupied MO, $\psi^{o}$. In the case of purely geometric projection through the space of molecular orbitals (both, occupied and virtual $), \boldsymbol{\psi}=\left(\boldsymbol{\psi}^{o}, \boldsymbol{\psi}^{v}\right)$, we get

$$
\left\langle\chi\left|\hat{P}_{\psi}^{o}+\hat{P}_{\psi}^{v}\right| \chi\right\rangle=\left\langle\chi\left|\left(\sum_{i=1}\left|\psi_{i}\right\rangle\left\langle\psi_{i}\right|\right)\right| \chi\right\rangle=\langle\chi \mid \chi\rangle=\mathbf{I}
$$

where the last equality results from the orthonormality relation in the orthogonalized AO representation.

\subsection{Electron population $N_{k}$}

The overall number of electrons in the molecule $N$ can be defined as a trace of the CBO matrix within the orthogonalized atomic orbital representation,

$$
N=\operatorname{tr}\{\boldsymbol{\gamma}\}=2 \sum_{k} \sum_{i}\left\langle\chi_{k} \mid \psi_{i}^{o}\right\rangle\left\langle\psi_{i}^{o} \mid \chi_{k}\right\rangle=2 \sum_{k} \sum_{i}\left|C_{k, i}\right|^{2}=\sum_{k} N_{k}
$$

Therefore, we interpret orbital population $N_{k}$ as an expectation value of operator $P_{\psi}^{o}$ in the state $\left|\chi_{k}\right\rangle$. Alternatively, one can also determine the overall number of electrons and orbital populations directly within the canonical molecular orbital representation. From the orthonormality of MO we obtain:

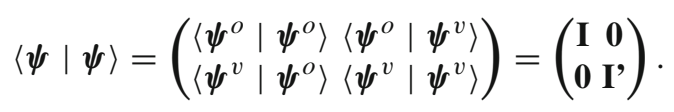

For the closed-shell molecular systems $N$ is determined as follows:

$$
N=2 \operatorname{tr}\left\{\left\langle\boldsymbol{\psi}^{o} \mid \boldsymbol{\psi}^{o}\right\rangle\right\}=2 \mathbf{I}=\mathbf{n}
$$

The overall number of electrons can be straightforwardly decomposed into orbital populations $\left\{N_{k}\right\}$ by introducing the orbital resolution of identity:

$$
N=2 \operatorname{tr}\left\{\left\langle\boldsymbol{\psi}^{o}\left|\left(\sum_{k}\left|\chi_{k}\right\rangle\left\langle\chi_{k}\right|\right)\right| \boldsymbol{\psi}^{o}\right\rangle\right\}=2 \sum_{k} \sum_{i}\left|C_{k, i}\right|^{2}=\sum_{k} N_{k} .
$$


Obviously, both decomposition schemes of overal number of electrons into orbital contributions, within AO and MO representations (Eqs. (6), (9), respectively) are fully equivalent.

\subsection{Covalency index $w_{k, l}$}

Most of the presently used quadratic bond orders [25-27] are related to the Wiberg covalency index between atom X and Y [28]:

$$
W_{X Y}=\frac{1}{2} \sum_{k}^{X} \sum_{l}^{Y} w_{k, l}=\frac{1}{2} \sum_{k}^{X} \sum_{l}^{Y}\left|\gamma_{k, l}\right|^{2} .
$$

According to Eq. (3) on can easily express each contribution $w_{k, l}$ to the Wiberg covalency index as an expectation value of the operator $S_{l} \equiv \hat{P}_{\psi}^{o} \hat{P}_{l} \hat{P}_{\psi}^{o}$ in the state $\left|\chi_{k}\right\rangle$ :

$$
w_{k, l}=4\left\langle\chi_{k}\left|\hat{P}_{\psi}^{o}\right| \chi_{l}\right\rangle\left\langle\chi_{l}\left|\hat{P}_{\psi}^{o}\right| \chi_{k}\right\rangle=4\left\langle\chi_{k}\left|\hat{S}_{l}\right| \chi_{k}\right\rangle
$$

Molecular scattering operator $\hat{S}_{\chi}$ arises within Orbital Communication Theory (OCT) [12] and has essential meaning in determining entropic bond descriptors of molecular communication systems. According to the OCT $w_{k, l}$ is an expectation value in the final (observable) state $\left|\chi_{k}\right\rangle$ of the molecular scattering operator $\hat{S}_{l}$ from the initial (reference) state $\left|\chi_{l}\right\rangle$, through the subspace of occupied molecular orbitals, $\psi^{o}$. In the information-theoretic sense the element $w_{k, l}$ determines amount of information about electrons assignment into atomic orbitals $\chi_{k}$ and $\chi_{l}$ that was dissipated (scattered) during bond forming proccess. In other words $-w_{k, l}$ measures amount of electrons that are delocalized between orbitals $\chi_{k}$ and $\chi_{l}$. Due to $\gamma$ is a hermitian matrix we automatically get $w_{k, l}=w_{l, k}$.

It follows directly from the idempotency properties of the density matrix $\boldsymbol{\gamma}$,

$$
(\boldsymbol{\gamma})^{2}=2 \boldsymbol{\gamma}, \quad \gamma_{k, k}=\frac{1}{2} \sum_{l}\left|\gamma_{k, l}\right|^{2},
$$

that for atomic orbital centered on atom $X$ the electron population $N_{k}$ can be decomposed into non-bonding (lone-pairs, core electrons) $N_{k}^{n}$ and bonding (chemical bonds) $N_{k}^{b}$ contributions:

$$
N_{k}=\frac{1}{2} \sum_{l} w_{k, l}=\frac{1}{2}\left(\sum_{l}^{X} w_{k, l}+\sum_{l}^{X^{c}} w_{k, l}\right)=N_{k}^{n}+N_{k}^{b},
$$

where $X^{c}$ complements the subspace of orthogonalized AOs centered on atom $X$. The same can be accomplished by introducing atomic resolution of identity, 


$$
1=\hat{S}_{X}+\left(1-\hat{S}_{X}\right)=\sum_{l}^{X} \hat{S}_{l}+\sum_{l}^{X^{c}} \hat{S}_{l}
$$

Then, according to Eq. (6) and using the following properties of operators $\hat{P}_{\psi}^{o}$ and $\hat{S}_{l}$ :

$$
\left(\hat{P}_{\psi}^{o}\right)^{2}=\hat{P}_{\psi}^{o}, \quad \hat{P}_{\psi}^{o}=\sum_{l} \hat{S}_{l}
$$

we immediately obtain

$$
N_{k}=2\left(\left\langle\chi_{k}\left|\hat{S}_{X}\right| \chi_{k}\right\rangle+\left\langle\chi_{k}\left|\left(1-\hat{S}_{X}\right)\right| \chi_{k}\right\rangle\right)=N_{k}^{n}+N_{k}^{b}
$$

Using again the atomic resolution of identity we can decompose $N_{k}^{b}$ further into particular bond order contributions:

$$
N_{k}^{b}=2\left\langle\chi_{k}\left|\left(1-\hat{S}_{X}\right)\right| \chi_{k}\right\rangle=2 \sum_{Y \neq X}\left\langle\chi_{k}\left|\hat{S}_{Y}\right| \chi_{k}\right\rangle
$$

Finally, for the closed-shell molecular systems we can define non-bonded electrons number, atomic valency (or rather atomic covalency) and bond covalency indices as simple functions of the molecular scattering operator in atomic resolution:

$$
\begin{gathered}
N_{X}^{n}=2 \operatorname{tr}_{X}\left\{\left\langle\chi\left|\hat{S}_{X}\right| \chi\right\rangle\right\}, \quad V_{X}=2 \operatorname{tr}_{X}\left\{\left\langle\chi\left|\left(1-\hat{S}_{X}\right)\right| \chi\right\rangle\right\} \\
W_{X Y}=2 \operatorname{tr}_{X}\left\{\left\langle\chi\left|\hat{S}_{Y}\right| \chi\right\rangle\right\}, \quad N_{X}=N_{X}^{n}+V_{X}=N_{X}^{n}+\sum_{X \neq Y} W_{X Y} .
\end{gathered}
$$

Alternatively, we can define Wiberg's covalency index $W_{X Y}$ as an expectation value of operator in the Hilbert space of canonical molecular orbitals. In analogy to preceding considerations we introduce another molecular scattering operator $\hat{S}_{X Y}$ originated from OCT and representing two sequentially connected molecular information channels:

$$
\hat{S}_{X Y}=\hat{S}_{X} \hat{S}_{Y}=\hat{P}_{\psi^{o}} \hat{P}_{X} \hat{P}_{\psi^{o}} \hat{P}_{Y} \hat{P}_{\psi^{o}}=\hat{P}_{\psi^{o}} \hat{M}_{X Y} \hat{P}_{\psi^{o}}
$$

Within Orbital Communication Theory the two-center projector $\hat{S}_{X Y}$ operates in the space of atomic orbitals and its expectation values reflect the influence of interacting atoms $X$ and $Y$ on particular atomic orbital. Moreover, using the one-center projector $\hat{S}_{X X}$ we can decompose the one-electron density matrix into two "layers", $\gamma^{n}$ and $\gamma^{b}$, describing non-bonding and bonding parts of the electron density, respectively: 


$$
\begin{aligned}
& \boldsymbol{\gamma}^{n}=2\left\langle\chi\left|\sum_{X} \hat{S}_{X X}\right| \chi\right\rangle, \quad N^{n}=\operatorname{tr}\left\{\boldsymbol{\gamma}^{n}\right\} \\
& \boldsymbol{\gamma}^{b}=2\left\langle\chi\left|\left(1-\sum_{X} \hat{S}_{X X}\right)\right| \chi\right\rangle, \quad N^{b}=\operatorname{tr}\left\{\boldsymbol{\gamma}^{b}\right\} .
\end{aligned}
$$

As opposed to $\hat{S}_{X Y}$ the projector $\hat{M}_{X Y}$ in Eq. (19) operates in the space of canonical molecular orbitals. The matrix of operator $\hat{M}_{X Y}$ within CMO basis is symmetric and can be straightforwardly partitioned into the following blocks:

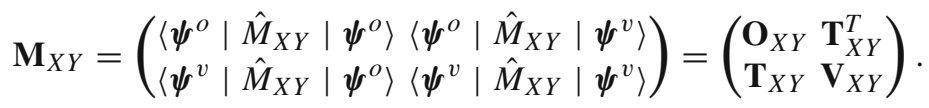

The expectation values of the operator $\hat{M}_{X Y}$ in the subspace of occupied molecular orbitals determine the effective contributions of each MO to bond order between atoms $X$ and $Y$ within the reference electron configuration of ground state $\left(\hat{P}_{\psi}^{o}\right)$. According to Eq. (3) we can express elements of $\mathbf{O}_{X Y}$ through the relevant elements of matrices LCAO MO and CBO:

$$
\mathbf{O}_{X Y}=\left\langle\boldsymbol{\psi}^{o}\left|\hat{M}_{X Y}\right| \boldsymbol{\psi}^{o}\right\rangle=\left\{\left(\mathbf{O}_{X Y}\right)_{i, j}=\sum_{k}^{X} \sum_{l}^{Y} C_{k, i}^{o} \gamma_{k, l} C_{l, j}^{o}\right\} .
$$

The $i$ th diagonal element of matrix $\mathbf{O}_{X Y}$ stands for the effective contribution of $i$ th canonical MO to the Wiberg index $W_{X Y}$ which in the case of closed-shell electron systems takes the form analogical to Eq. (2):

$$
W_{X Y}=\operatorname{tr}\left\{\mathbf{O}_{X Y}\right\}=\sum_{i}^{o c c} O_{i, i} \equiv \sum_{i}^{o c c} W_{X Y}^{(i)}
$$

It is typical for canonical molecular orbitals which are delocalized over the whole molecule that the off-diagonal elements of matrix $\mathbf{O}_{X Y}$ assume non-zero values. In particular, $\left(\mathbf{O}_{X Y}\right)_{i, j}<0$ means that molecular orbitals $\psi_{i}$ and $\psi_{j}$ are out of phase with respect to chemical bond $\mathrm{X}-\mathrm{Y}$, whereas $\left(\mathbf{O}_{X Y}\right)_{i, j}>0$ reflects its phase-coherence. The case $\left(\mathbf{O}_{X Y}\right)_{i, j}=0$ in turn reflects the fact that the occupation of orbital $\psi_{i}$ does not affect the contribution of orbital $\psi_{j}$ to the bond order $W_{X Y}$. The matrix of the operator $\hat{M}_{X Y}$ within the subspace of virtual molecular orbitals reveals similar properties:

$$
\mathbf{V}_{X Y}=\left\{\left(V_{X Y}\right)_{i, j}=\sum_{k}^{X} \sum_{k}^{Y} C_{k, i}^{v} \gamma_{k, l} C_{l, j}^{v}\right\}, \quad \operatorname{tr}\left\{\mathbf{V}_{X Y}\right\}=-W_{X Y}
$$

In analogy to Eqs. (22) and (23) the number of electrons assigned to atom $X$ and not contributing to the valence index $V_{X}$ can be defined as a trace of matrix of the operator $\hat{M}_{X X}$ in the subspace of occupied MO: 


$$
\mathbf{O}_{X X}=\left\{\left(\mathbf{O}_{X Y}\right)_{i, j}=\sum_{k}^{X} \sum_{l}^{X} C_{k, i}^{o} \gamma_{k, l} C_{l, j}^{o}\right\}, \quad \operatorname{tr}\left\{\mathbf{O}_{X X}\right\}=N_{X}^{b}
$$

\section{Noiseless MO-channel: the eigenproblem of $\hat{M}_{X Y}$}

Within the Communication Theory of the Chemical Bond the operator $\hat{M}_{X Y}$ represents the resultant information cascade [12] in which the information about MO contribution to particular chemical bond (or molecular fragment) is transmitted. For stationary electron distribitions [12,29] one can discern two extreme cases of MO-channel's transmission (see Fig. 1) [30]:

- stochastic - typical for molecular communication systems with all MOs mutually communicated, i.e. with non-zeroed both, diagonal and off-diagonal elements of matrix of conditional-probability amplitudes (21),

- fully deterministic and noiseless - characteristic for information channels defined by unit matrix of the relevant conditional probabilities (off-diagonal communications zeroed).

In the whole-molecule resolution the relevant matrix (21) is the identity matrix, i.e. transmission of information about electron distribution between $\left\{\psi_{i}\right\}$ is noiseless and deterministic. However, since canonical molecular orbitals are delocalized over the whole molecule, in the diatomic $\mathrm{X}-\mathrm{Y}$ resolution some noise appears in the same transmission. The natural representation of localized orbitals forming a noiseless (and deterministic) communication system can be reached by finding representation for which the matrix of the operator $\hat{M}_{X Y}$ takes a diagonal form.

Hence, diagonalization of matrix of the operator $\hat{M}_{X Y}$ within the space of all molecular orbitals (occupied and virtual), which is somehow similar to diagonalization of two-center atomic blocks of the density matrix $\gamma$ [10], gives rise to alternative bond order orbitals (BOO) representation. As proved by Jug [10], the classical bond order orbitals appear in pairs with the opposite signs of its eigenvalues and vanishing eigenvalues one observes always when the number basis functions on each of the two atoms

Stochastic channel

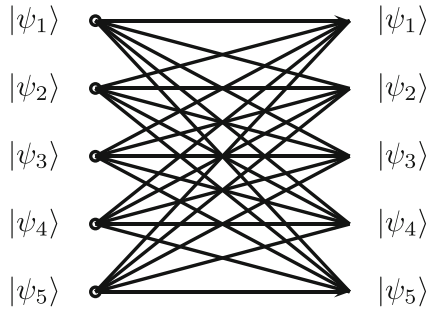

$\mathbf{M}=\left\{M_{i, j} \geq 0\right\}$
Deterministic channel (noiseless)

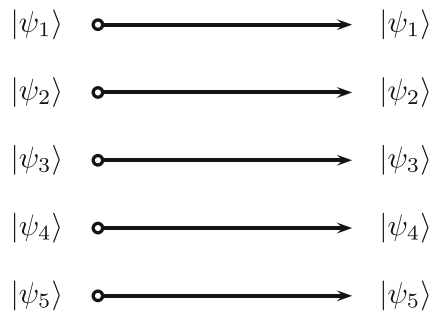

$$
\mathbf{M}=\left\{M_{i, j}=\delta_{i, j}\right\}
$$

Fig. 1 Two extreme cases of stationary communication systems defined within 5-dimentional Hilbert space of states $\left\{\left|\psi_{i}\right\rangle\right\}$ 
$X$ and $Y$ is different. The sum of all eigenvalues is equal to zero and the overall bond order can be obtained using the Mulliken overlap criterion [10] or-alternatively, the vector projection weighting procedure [31].

Due to natural partition of Hilbert space of molecular orbitals onto occupied and virtual MO subspaces for closed-shell electron configurations, introducing the operator $\hat{M}_{X Y}$ allows one to consider independently two eigenproblems. By diagonalization of matrix $\mathbf{O}_{X Y}$ we obtain the spectrum of eigenvalues that sum up to the bond order $W_{X Y}$ :

$$
\sum_{s} \lambda_{s}^{b}=\operatorname{tr}\left\{\lambda^{b}\right\}=\operatorname{tr}\left\{\mathbf{U}_{b}^{-1} \mathbf{O}_{X Y} \mathbf{U}_{b}\right\}=\operatorname{tr}\left\{\mathbf{O}_{X Y}\right\}=W_{X Y}
$$

and determine occupations of the bonding subset of bond order orbitals of the chemical bond $\mathrm{X}-\mathrm{Y}$ (the eigenvectors of matrix $\mathbf{O}_{X Y}$ ),

$$
\begin{aligned}
B O O \equiv \boldsymbol{\phi}^{b} & =\boldsymbol{\psi}^{o} \mathbf{U}_{b}=\chi \mathbf{C}^{o} \mathbf{U}_{b}=\chi \mathbf{C}^{b}, \\
\phi_{s}^{b} & =C_{1, s}^{b} \chi_{1}+C_{2, s}^{b} \psi_{2}+\ldots+C_{n, s}^{b} \chi_{n} .
\end{aligned}
$$

In turn, the sum of eigenvalues of the operator $\hat{M}_{X Y}$ within the subspace $\psi^{v}$ reads

$$
\sum_{s^{\prime}} \lambda_{s^{\prime}}^{a}=\operatorname{tr}\left\{\lambda^{a}\right\}=\operatorname{tr}\left\{\mathbf{U}_{a}^{-1} \mathbf{V}_{X Y} \mathbf{U}_{a}\right\}=\operatorname{tr}\left\{\mathbf{V}_{X Y}\right\}=-W_{X Y}
$$

and given eigenvectors determine a subset of anti-bonding $\mathrm{BOO}^{*}$ :

$$
\begin{aligned}
B O O^{*} \equiv \boldsymbol{\phi}^{a} & =\boldsymbol{\psi}^{v} \mathbf{U}_{v}=\chi \mathbf{C}^{v} \mathbf{U}_{a}=\chi \mathbf{C}^{a}, \\
\phi_{s^{\prime}}^{a} & =C_{n+1, s^{\prime}}^{a} \chi_{n+1}+C_{n+2, s^{\prime}}^{a} \chi_{n+2}+\ldots+C_{n+m, s^{\prime}}^{a} \chi_{n+m} .
\end{aligned}
$$

In general, due to non-zero elements in matrix $\mathbf{T}_{X Y}$ the eigenvalue spectrum $\lambda$ of the operator $\mathbf{M}_{X Y}$ differs from eigenvalus $\lambda^{b}$ and $\lambda^{a}$. However, it fulfills the following relation:

$$
\sum_{s} \lambda_{s}=\operatorname{tr}\{\lambda\}=\operatorname{tr}\left\{\mathbf{M}_{X Y}\right\} \equiv \operatorname{tr}\left\{\mathbf{O}_{X Y}\right\}+\operatorname{tr}\left\{\mathbf{V}_{X Y}\right\}=0
$$

Similarly, solving the eigenproblem of the operator $\hat{M}_{X X}$ within the subspace of occupied MO gives rise to the spectrum of eigenvalues $\lambda^{n}$ that sum up to the overall number of electrons assigned to atom $X$ and not contributing to the valence index $V_{X}$

$$
\sum_{s} \lambda_{s}^{n}=\operatorname{tr}\left\{\lambda^{n}\right\}=\operatorname{tr}\left\{\mathbf{U}_{n}^{-1} \mathbf{O}_{X X} \mathbf{U}_{n}\right\}=\operatorname{tr}\left\{\mathbf{O}_{X X}\right\}=N_{X}^{n} .
$$

In general, eigenvalues close to 2 identify core electrons and lone-pairs while the rest of non-zero eigenvalues are usually connected with the charge transfer effect and are a distinctive feature of molecular systems with polarized chemical bonds. 
Further, let us consider the case of non-typical electron deficient chemical bond a three-center two-electron (3c2e) bond, X-Y-Z, where two electrons are delocalized over three atoms $X, Y$ and $Z$. Orbital Communication Theory allows one to define a relevant molecular communication system in which transmission of information about electrons shared by atoms $Y$ and $Z$ is conditioned by information about electrons between atoms $Y$ and $Z$. Entropic descriptors of such cascade model of sequentially connected information subchannels have already been the subject of investigation $[12,32,33]$. In this paper we consider a model of parallely connected information subchannels which constitutes an alternative for the former one. The parallel connection of communication subsystems means that transmissions of information about electrons shared in bonds $\mathrm{Y}-\mathrm{Z}$ and $\mathrm{X}-\mathrm{Y}$ are not mutually conditioned. In such a case the three-center scattering operator is simply defined as a sum of relevant two-center operators,

$$
\hat{S}_{X Y Z}=\hat{S}_{X Y}+\hat{S}_{Y Z}=\hat{P}_{\psi}\left(\hat{M}_{X Y}+\hat{M}_{Y Z}\right) \hat{P}_{\psi}=\hat{P}_{\psi} \hat{M}_{X Y Z} \hat{P}_{\psi}
$$

and matrix of the operator $\hat{M}_{X Y Z}$ in the subspace of occupied MO reads

$$
\mathbf{O}_{X Y Z}=\left\langle\boldsymbol{\psi}^{o}\left|\hat{M}_{X Y Z}\right| \boldsymbol{\psi}^{o}\right\rangle=\mathbf{O}_{X Y}+\mathbf{O}_{Y Z}
$$

We have to notice here that in the adopted definition of $\hat{S}_{X Y Z}$ we do not include direct communication (through-space interaction) between atoms $\mathrm{X}$ and $\mathrm{Z}$, represented by $\hat{S}_{X Z \text {. }}$

Summation of $\hat{M}_{X Y}$ over all chemical bonds in the molecule leads to

$$
\hat{M}=\sum_{X} \sum_{Y \neq X} \hat{M}_{X Y}=2\left(1-\sum_{X} \hat{M}_{X X}\right) .
$$

By separation of non-interacting electrons of inner shells in the case of molecule with purely covalent chemical bonds and in the absence of lone-pairs the sum of one-center operators in the preceding equation vanishes and matrix $\mathbf{O}_{X Y Z}$ assumes the following diagonal form:

$$
\mathbf{O}_{X Y Z}=\left(\begin{array}{cc}
\mathbf{0}^{\text {core }} & \mathbf{0} \\
\mathbf{0} & \mathbf{2}^{\text {val }}
\end{array}\right)
$$

In such a case $\mathbf{O}_{X Y Z} \equiv \lambda^{b}$ and according to Eqs. (26) and (27) we have $\boldsymbol{\phi}^{b} \equiv$ $\psi^{\circ}$. Therefore, each canonical molecular orbital of the valence shell of the $n$-atomic molecule with the closed-shell electron configuration in a sense can be regarded as a bond order orbital of a $n$-center-2-electron chemical bond. 


\section{Numerical results}

To examine how the proposed bond-order decomposition procedure manages with several representative types of chemical bonds we used wavefunctions calculated at RHF as well as DFT/B3LYP [34] theory levels within MINI [35] and cc-pVDZ [36] basis sets, respectively, using the standard ab initio quantum chemistry package, Gamess [37]. The bond order orbitals were generated by special program written by authors and visualized (after deorthogonalization) using the molecular visualization program, Molekel [38].

In the first place eigenvalues from diagonalization of one- and two-center blocks of the density matrix of water molecule were compared with eigenvalues of operator $\hat{M}_{X Y}$ within canonical molecular orbitals space (both, occupied and virtual). Table 1 contains the results obtained from RHF/MINI calculations. As we can see, both decomposition schemes appropriately identify core electrons and lone-pairs $\left(\lambda_{1-3}=2\right.$ for oxygen atom) and give rise to similar eigenvalues for chemical bond $\mathrm{O}-\mathrm{H}$. The main differences concern the eigenvalues $\lambda_{4}$ and $\lambda_{5}$ related to contribution of oxygen atom into chemical bonds: the former method allows one to count the overall electron population while the latter method does not include the overlap populations and thus non-zero eigenvalues indicate only the number of electrons well localized on the oxygen atom. Also, in accordance to expectations, eigenvalues of the operator $\hat{M}_{X Y}$ associated with bonding and anti-bonding BOOs were calculated separately which was not the case for eigenvalues of the two-center atomic blocks of the density matrix. All bond order orbitals from the newly proposed definition as well as eigenvectors of matrix $\mathbf{O}_{O O}$ were visualized and are presented in Fig. 2. One can observe that our method not only reliably reproduces qualitative picture of the electronic structure of water molecule obtained using the Pipek-Mezey's MO-localization procedure but also provides the relevant anti-bonding orbitals.

Table 2, in turn, presents bond orders $W_{X Y}$ and their $\sigma-, \pi$-components, $W_{X Y}^{\sigma}$ and $W_{X Y}^{\pi}=W_{X Y}^{\pi^{1}}+W_{X Y}^{\pi^{2}}$ respectively, determined by the relevant eigenvectors of $\mathbf{O}_{X Y}$

Table 1 Comparison of eigenvalues from diagonalization of two-atomic blocks of matrix $\gamma$ and eigenvalues of operator $\hat{M}_{X Y}$ within CMO space for water molecule

\begin{tabular}{|c|c|c|c|c|c|c|}
\hline \multirow[t]{2}{*}{ Matrix } & \multirow[t]{2}{*}{ Atom/bond } & \multicolumn{5}{|c|}{ Eigenvalues } \\
\hline & & $\lambda_{1}$ & $\lambda_{2}$ & $\lambda_{3}$ & $\lambda_{4}$ & $\lambda_{5}$ \\
\hline$\gamma$ & $\mathrm{O}$ & 2.00 & 2.00 & 2.00 & 1.23 & 1.20 \\
\hline$\gamma$ & $\mathrm{H}$ & 0.79 & 0.00 & 0.00 & 0.00 & 0.00 \\
\hline$\gamma$ & $\mathrm{O}-\mathrm{H}$ & 0.98 & -0.98 & 0.00 & 0.00 & 0.00 \\
\hline $\mathbf{O}_{X}$ & $\mathrm{O}$ & 2.00 & 2.00 & 2.00 & 0.38 & 0.36 \\
\hline $\mathbf{O}_{X}$ & $\mathrm{H}$ & 0.31 & 0.00 & 0.00 & 0.00 & 0.00 \\
\hline $\mathbf{O}_{X Y}$ & $\mathrm{O}-\mathrm{H}$ & 0.96 & 0.00 & 0.00 & 0.00 & 0.00 \\
\hline $\mathbf{v}_{X Y}$ & $\mathrm{O}-\mathrm{H}$ & -0.96 & 0.00 & 0.00 & 0.00 & 0.00 \\
\hline
\end{tabular}

Method: RHF/MINI 


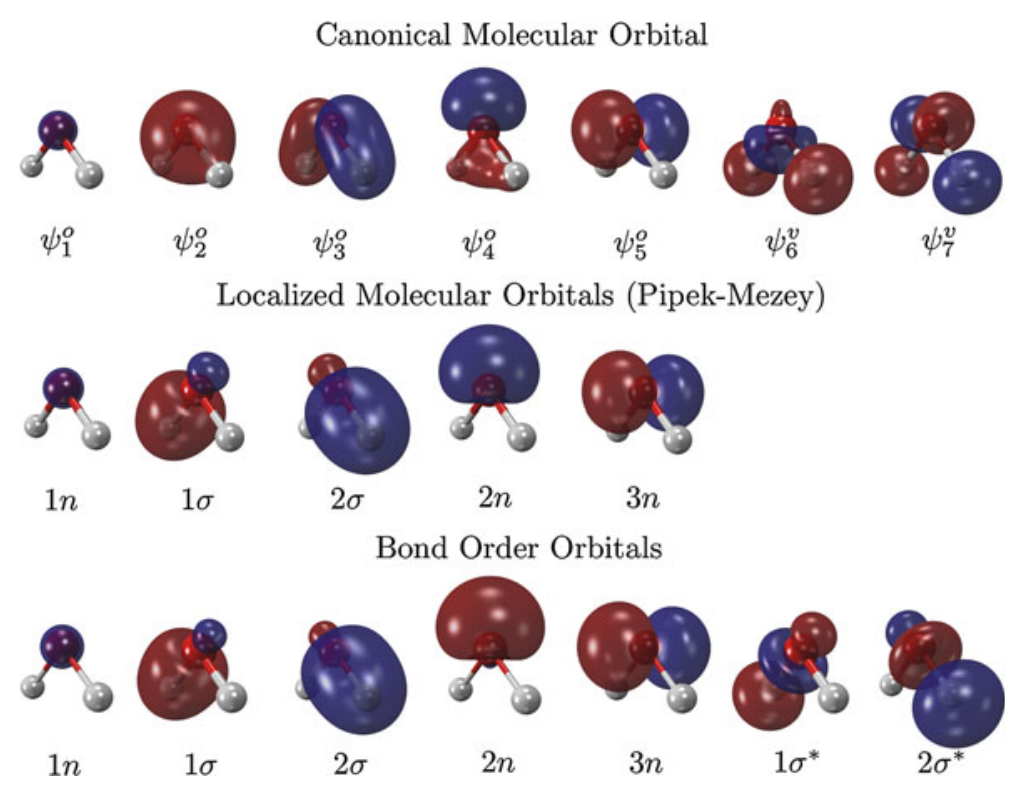

Fig. 2 Comparison of canonical molecular orbitals (CMO) with localized molecular orbitals (LMO) from Pipek-Mezey's procedure and bond order orbitals (BOO) from presently indtroduced BOO-resolved decomposition of chemical bond order. Method: RHF/MINI

and calculated using two different methods: RHF/MINI and B3LYP/cc-pVDZ. For comparison, also three different definitions of quadratic bond orders were used:

- Wiberg-type bond orders calculated within Löwdin-orthogonalized atomic orbital representation [22],

- Wiberg-type bond orders calculated within "physically"-orthogonalized atomic orbital representation, recently proposed by the authors [39],

- Mayer's bond orders [3,25] calculated within non-orthogonal atomic orbital representation. In this particular case the appropriate elements of matrix $\mathbf{O}_{X Y}$ are defined as follows:

$$
\left(\mathbf{O}_{X Y}\right)_{m, n}=\sum_{k}^{X} \sum_{l}^{Y}\left(\mathbf{C}^{o} \mathbf{C}^{o \dagger} \mathbf{S}\right)_{k, l}\left(\mathbf{c}_{n}^{o} \mathbf{c}_{m}^{o \dagger} \mathbf{S}\right)_{l, k}
$$

where $\mathbf{c}_{n}$ and $\mathbf{c}_{n}$ are the relevant columns of the LCAO matrix.

The component $W_{X Y}^{r}$ stands for the sum of all eigenvalues that represent neither $\sigma$ nor $\pi$ chemical bonds and usually have quite small values. Thus, $W_{X Y}^{r} \neq 0$ may indicate a correction from the outer hybrids (e.g. in the case of conjugated $\pi$ bonds), anti-bonding orbitals, orthogonalization artemaths, etc.. At the first glance we can observe in Table 2 that decomposition of Mayer's bond orders leads to very large values of $W_{X Y}^{r}$ as well as $\sigma$-eigenvalues that remarkably exceed 1 which practically disqualify this variant of BO-decomposition. Using the orthogonalized basis sets ensures the appropriate eigenvalues of $\sigma$-components, however $W_{X Y}^{r}$ can still 
Table 2 Comparison of Wiberg-type bond orders $W_{X Y}$ and its components $W_{X Y}^{\sigma}, W_{X Y}^{\pi}$ and $W_{X Y}^{r}$ from BOO-resolved decomposition scheme for several molecules at two different computational levels: RHF/MINI and B3LYP/cc-pVDZ

\begin{tabular}{|c|c|c|c|c|c|c|c|c|}
\hline \multirow[t]{2}{*}{ Chemical bond } & \multicolumn{4}{|c|}{ RHF / MINI } & \multicolumn{4}{|c|}{ B3LYP / cc-pVDZ } \\
\hline & $W_{X Y}$ & $W_{X Y}^{\sigma}$ & $W_{X Y}^{\pi}$ & $W_{X Y}^{r}$ & $W_{X Y}$ & $W_{X Y}^{\sigma}$ & $W_{X Y}^{\pi}$ & $W_{X Y}^{r}$ \\
\hline \multicolumn{9}{|c|}{ Wiberg's bond orders calculated within "geometrically" orthogonalized AO representation } \\
\hline $\mathrm{N}_{2}$ & 3.00 & 1.00 & 2.00 & 0.00 & 3.42 & 1.00 & 2.00 & 0.42 \\
\hline $\mathrm{HF}$ & 0.92 & 0.92 & 0.00 & 0.00 & 1.21 & 0.91 & 0.00 & 0.30 \\
\hline $\mathrm{CO}$ & 2.49 & 0.95 & 1.54 & 0.00 & 3.06 & 0.96 & 1.72 & 0.38 \\
\hline $\mathrm{NaCl}$ & 0.46 & 0.46 & 0.00 & 0.00 & 1.37 & 0.60 & 0.00 & 0.77 \\
\hline $\mathrm{C}_{2} \mathrm{H}_{6}(\mathrm{C}-\mathrm{C})$ & 1.14 & 1.00 & 0.00 & 0.14 & 1.22 & 0.92 & 0.00 & 0.30 \\
\hline $\mathrm{C}_{2} \mathrm{H}_{4}(\mathrm{C}-\mathrm{C})$ & 2.13 & 1.00 & 1.00 & 0.13 & 2.17 & 0.93 & 0.95 & 0.29 \\
\hline $\mathrm{C}_{2} \mathrm{H}_{2}(\mathrm{C}-\mathrm{C})$ & 3.03 & 1.00 & 2.00 & 0.03 & 3.09 & 0.97 & 1.92 & 0.20 \\
\hline $\mathrm{C}_{6} \mathrm{H}_{6}(\mathrm{C}-\mathrm{C})$ & 1.64 & 0.98 & 0.56 & 0.10 & 1.63 & 0.90 & 0.52 & 0.21 \\
\hline \multicolumn{9}{|c|}{ Wiberg's bond orders calculated within "physically" orthogonalized AO representation } \\
\hline $\mathrm{N}_{2}$ & 3.00 & 1.00 & 2.00 & 0.00 & 3.00 & 1.00 & 2.00 & 0.00 \\
\hline $\mathrm{HF}$ & 0.79 & 0.79 & 0.00 & 0.00 & 0.89 & 0.88 & 0.00 & 0.01 \\
\hline $\mathrm{CO}$ & 2.03 & 0.75 & 1.28 & 0.00 & 2.29 & 0.87 & 1.42 & 0.00 \\
\hline $\mathrm{NaCl}$ & 0.25 & 0.25 & 0.00 & 0.00 & 0.31 & 0.18 & 0.00 & 0.13 \\
\hline $\mathrm{C}_{2} \mathrm{H}_{6}(\mathrm{C}-\mathrm{C})$ & 0.96 & 0.96 & 0.00 & 0.00 & 1.00 & 0.95 & 0.00 & 0.05 \\
\hline $\mathrm{C}_{2} \mathrm{H}_{4}(\mathrm{C}-\mathrm{C})$ & 1.98 & 0.98 & 1.00 & 0.00 & 2.03 & 0.95 & 1.00 & 0.08 \\
\hline $\mathrm{C}_{2} \mathrm{H}_{2}(\mathrm{C}-\mathrm{C})$ & 3.00 & 1.00 & 2.00 & 0.00 & 2.71 & 0.73 & 2.00 & -0.02 \\
\hline $\mathrm{C}_{6} \mathrm{H}_{6}(\mathrm{C}-\mathrm{C})$ & 1.41 & 0.96 & 0.56 & -0.11 & 1.33 & 0.85 & 0.55 & -0.07 \\
\hline \multicolumn{9}{|c|}{ Mayer's bond orders calculated within non-orthogonal AO representation } \\
\hline $\mathrm{N}_{2}$ & 3.00 & 1.42 & 2.00 & -0.42 & 2.93 & 1.32 & 2.00 & -0.39 \\
\hline $\mathrm{HF}$ & 0.86 & 1.13 & 0.00 & -0.27 & 1.04 & 1.17 & 0.00 & -0.13 \\
\hline $\mathrm{CO}$ & 2.38 & 1.29 & 1.50 & -0.41 & 2.62 & 1.22 & 1.64 & -0.24 \\
\hline $\mathrm{NaCl}$ & 0.38 & 0.50 & 0.00 & -0.12 & 0.86 & 0.52 & 0.00 & 0.34 \\
\hline $\mathrm{C}_{2} \mathrm{H}_{6}(\mathrm{C}-\mathrm{C})$ & 1.02 & 1.27 & 0.00 & -0.25 & 1.12 & 1.29 & 0.00 & -0.17 \\
\hline $\mathrm{C}_{2} \mathrm{H}_{4}(\mathrm{C}-\mathrm{C})$ & 2.03 & 1.28 & 1.00 & -0.25 & 2.13 & 1.26 & 0.98 & -0.11 \\
\hline $\mathrm{C}_{2} \mathrm{H}_{2}(\mathrm{C}-\mathrm{C})$ & 3.01 & 1.14 & 2.00 & -0.13 & 2.71 & 1.24 & 1.98 & -0.51 \\
\hline $\mathrm{C}_{6} \mathrm{H}_{6}(\mathrm{C}-\mathrm{C})$ & 1.45 & 1.32 & 0.60 & -0.47 & 1.46 & 1.26 & 0.60 & -0.40 \\
\hline
\end{tabular}

Three alternative definitions of bond orders were used (more details in the text)

assume significant non-zero values. Particularly, the overestimated values of Wibergtype bond orders defined within Löwdin-orthogonalized extended (but also minimal) basis set are determined in the greater part by large $W_{X Y}^{r}$ values, even for diatomics. A comparison with the relevant values calculated using "physical" orthogonalization procedure allows one to draw a conclusion that the "geometrical" (involving all canonical molecular orbitals regardless of its occupations [39]) orthogonalization originally proposed by Löwdin significantly affects the quadratic bond-order indices with contributions that do not represent any of pure bond components. Thus, using the recently proposed by the authors orthogonalization procedure seems to be the most suitable 
Table 3 Comparison of BO-components: $W_{X Y}^{\sigma}, W_{X Y}^{\pi}$ and $W_{X Y}^{r}$ from BOO-resolved decomposition scheme for several cycloalkenes and its aromatic equivalents

\begin{tabular}{lllllllr}
\hline Molecule & $W_{\mathrm{C}=\mathrm{C}}^{\sigma}$ & $W_{\mathrm{C}=\mathrm{C}}^{\pi}$ & $W_{\mathrm{C}=\mathrm{C}}^{r}$ & Molecule & $W_{\mathrm{C}=\mathrm{C}}^{\sigma}$ & $W_{\mathrm{C}=\mathrm{C}}^{\pi}$ & $W_{\mathrm{C}=\mathrm{C}}^{r}$ \\
\hline $\mathrm{C}_{3} \mathrm{H}_{4}$ & 0.98 & 0.96 & 0.03 & $\mathrm{C}_{3} \mathrm{H}_{3}^{+}$ & 0.96 & 0.45 & 0.00 \\
$\mathrm{C}_{4} \mathrm{H}_{4}$ & 0.95 & 1.00 & 0.00 & $\mathrm{C}_{4} \mathrm{H}_{4}^{2-}$ & 0.94 & 0.50 & -0.26 \\
$\mathrm{C}_{5} \mathrm{H}_{6}$ & 0.96 & 0.93 & -0.01 & $\mathrm{C}_{5} \mathrm{H}_{5}^{-}$ & 0.96 & 0.60 & -0.19 \\
$\mathrm{C}_{6} \mathrm{H}_{8}$ & 0.97 & 0.94 & -0.01 & $\mathrm{C}_{6} \mathrm{H}_{6}$ & 0.96 & 0.56 & -0.11 \\
$\mathrm{C}_{7} \mathrm{H}_{8}$ & 0.97 & 0.93 & -0.02 & $\mathrm{C}_{7} \mathrm{H}_{7}^{+}$ & 0.96 & 0.48 & -0.07 \\
$\mathrm{C}_{8} \mathrm{H}_{8}$ & 0.97 & 0.95 & -0.01 & $\mathrm{C}_{8} \mathrm{H}_{8}^{2-}$ & 0.96 & 0.56 & -0.20 \\
$\mathrm{C}_{9} \mathrm{H}_{10}$ & 0.97 & 0.95 & -0.01 & $\mathrm{C}_{9} \mathrm{H}_{10}^{-}$ & 0.96 & 0.56 & -0.15 \\
$\mathrm{C}_{10} \mathrm{H}_{10}$ & 0.97 & 0.94 & -0.01 & $\mathrm{C}_{10} \mathrm{H}_{8}$ & 0.96 & 0.51 & -0.12 \\
\hline
\end{tabular}

Method: RHF/MINI ("physically" orthogonalized AO)

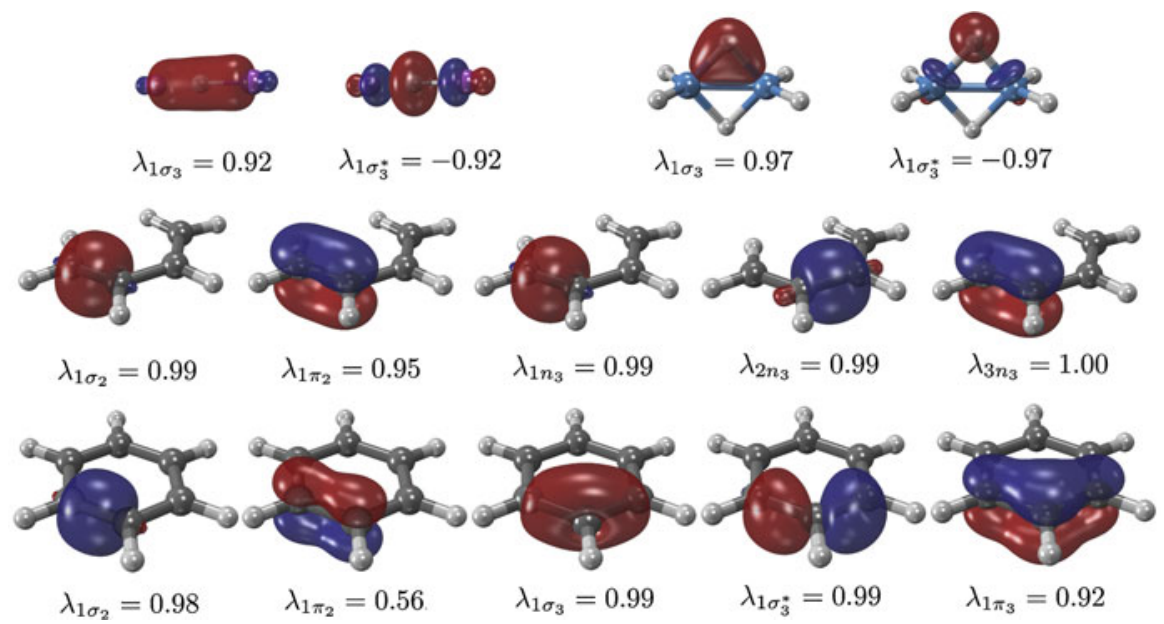

Fig. 3 Bond order orbitals and its occupations obtained from diagonalization of matrices $\mathbf{O}_{X Y Z}$ and $\mathbf{V}_{X Y Z}$ for typical three-center two-electron molecular systems: $\mathrm{HF}_{2}^{-}$anion and diborane $\mathrm{B}_{2} \mathrm{H}_{6}$ molecule, and for systems without/with $\pi$-delocalization: 1,3-butadiene $\mathrm{C}_{4} \mathrm{H}_{6}$ and benzene $\mathrm{C}_{6} \mathrm{H}_{6}$. Method RHF/MINI (Löwdin-orthogonalized AOs)

for bond order analysis and all further calculations presented in this paper involve the "physically"-orthogonalized AO-representation.

It follows from Table 2 that in minimal basis set the majority of $W_{X Y}^{r}$ indices assume negligible values with the exception of benzene molecule with significant negative value that could be connected with the presence of system of $\pi$-conjugated chemical bonds. To investigate the effect of electron delocalization on BO-decomposition we carried out the calculations of bond components for several representative cycloalkenes and its aromatic equivalents and results are collected in Table 3. Even a cursory analysis of $W_{X Y}^{r}$ values in Table 3 fully confirms that the presence of $\pi$-conjugated chemical bonds in the molecule remarkably increases the number of small negative eigenvalues 
and consequently $W_{X Y}^{r}<0$. One can also observe that $W_{X Y}^{r}$ well correlate with the number of delocalized electrons per carbon atom in the ring.

Finally, it was of our interest to study bond order orbitals of 3-center 2-electron chemical bonds. BOO and BOO* were calculated at RHF/MINI theory level for selected $\mathrm{X}-\mathrm{Y}-\mathrm{Z}$ configurations of atoms in the following molecules: $\mathrm{HF}_{2}^{-}(\mathrm{F}-\mathrm{H}-\mathrm{F})$, $\mathrm{B}_{2} \mathrm{H}_{6}(\mathrm{~B}-\mathrm{H}-\mathrm{B}), \mathrm{C}_{4} \mathrm{H}_{6}\left(\mathrm{C}_{1}=\mathrm{C}_{2}-\mathrm{C}_{3}\right)$ and $\mathrm{C}_{6} \mathrm{H}_{6}\left(\mathrm{C}_{1}-\mathrm{C}_{2}-\mathrm{C}_{3}\right)$ and results are presented in Figure 3. As we can see, eigenvectors of matrices $\mathbf{O}_{X Y Z}$ and $\mathbf{V}_{X Y Z}$ reliably identify the 3-center bond order orbitals in bifluoride anion and diborane molecule. The associated eigenvalues of $\mathrm{BOO}$ and $\mathrm{BOO}^{*}\left(\lambda_{1 \sigma_{3}}\right.$ and $\lambda_{1 \sigma_{3}^{*}}$, respectively) in both species indicate a single, covalent bonds which is in agreement with chemical intuition.

Three-center bond order orbitals for 1,3-butadiene and benzene molecules in turn reveal some features that seem to be essential for distinguishing between bonds with and without electron delocalization. Namely, in the case of benzene molecule for each set of adjacent three carbon atoms there are always three bond order orbitals: pair of equally occupied $1 \sigma_{3}$ and $1 \sigma_{3}^{*}$ which give rise to the vanishing $\sigma$-density, and one occupied $1 \pi_{3}$ orbital which determines the resultant $3 \mathrm{c} 2 \mathrm{e}$ chemical bond of $\pi$-type. As opposed to results for $\mathrm{C}_{6} \mathrm{H}_{6}$ molecule decomposition of the three-center bonds in $\mathrm{C}_{4} \mathrm{H}_{6}$ provides completely different set of bond order orbitals: a pair of non-bonding (with respect to all three atoms) $1 n_{3}$ and $2 n_{3}$ orbitals accurately reproducing the relevant two-center BOOs, $1 \sigma_{2}$ and $2 \sigma_{2}$. The third bond order orbital is also non-bonding with respect to $\mathrm{C}_{1}=\mathrm{C}_{2}-\mathrm{C}_{3}$ bond but it is very similar to the relevant non-conjugated $1 \pi_{2}$ orbital.

\section{Conclusions}

In this paper we have proposed a scheme of decomposing chemical bond into anew defined the bond order orbitals which constitute an alternative for those originally defined by Jug. Solving the eigenproblem of the scattering operator, originated from the orbital communication theory, allows one to tackle the problem of two- and threecenter bond analysis within the same framework. The new definitions give rise to alternative interpretation of molecular orbital of the valence shell in the case of $n$-atomic molecule (closed-shell electron configuration) as a bond order orbital of $n$-center-2electron chemical bond. The calculations we have carried out clearly show that the new method of bond order decomposition properly copes with identifying the relevant bond components $(\sigma, \pi$, etc..). An analysis of numerical results brings to light the fact that the most transparent and reliable picture of the chemical bond structure can be obtained within the minimal basis set of "physically" orthogonalized atomic orbitals.

The chemical bond decomposition involving the eigenproblem of the appropriate scattering operator within the orthogonalized MO space is still need of thorough examination and this study is currently in progress.

Acknowledgments This work was supported by the T-Donation for Young Scientists and PhD Students, Grant No. PSP: K/DSC/000133 (from Department of Chemistry, Jagiellonian University).

Open Access This article is distributed under the terms of the Creative Commons Attribution License which permits any use, distribution, and reproduction in any medium, provided the original author(s) and the source are credited. 


\section{References}

1. L. Pauling, J. Am. Chem. Soc. 69, 542 (1947)

2. N.P. Borisova, S.G. Semenov, Vestn. Lenin. Univ. 16, 119 (1973)

3. I. Mayer, J. Comput. Chem. 28, 204 (2007)

4. S.F. Boys, Rev. Mod. Phys. 32, 296 (1960)

5. C. Edmiston, K. Ruedenberg, J. Chem. Phys. 43, S97 (1965)

6. J. Pipek, P.G. Mezey, J. Chem. Phys. 90, 4916 (1989)

7. A.E. Reed, L.A. Curtiss, F. Weinhold, Chem. Rev. 88, 899 (1988)

8. A. Michalak, M. Mitoraj, T. Ziegler, J. Phys. Chem. 2008, 112 (1933)

9. O.V. Sizova, L.V. Skripnikov, A.Y. Sokolov, V.V. Sizov, Int. J. Quant. Chem. 109, 2581 (2009)

10. K. Jug, J. Am. Chem. 99, 7800 (1977)

11. R.F. Nalewajski, Information Theory of the Molecular Systems (Elsevier, Amsterdam, 2006)

12. R.F. Nalewajski, Information Origins of the Chemical Bond (Nova Science, Hauppauge, NY, 2010)

13. R.F. Nalewajski, Perspectives in Electronic Structure Theory (Springer, Heidelberg, 2012)

14. R.F. Nalewajski, Phys. Chem. Chem. Phys. 4, 4952 (2002)

15. R.F. Nalewajski, Int. J. Quant. Chem. 109, 425 (2009)

16. R.F. Nalewajski, Int. J. Quant. Chem. 109, 2495 (2009)

17. R.F. Nalewajski, Adv. Q. Chem. 58, 217 (2009)

18. R.F. Nalewajski, J. Math. Chem. 49, 2308 (2011)

19. R.F. Nalewajski, D. Szczepanik, J. Mrozek, Adv. Q. Chem. 61, 1 (2011)

20. R.F. Nalewajski, D. Szczepanik, J. Mrozek, J. Math. Chem. 50, 1437 (2012)

21. C.A. Coulson, Proc. R. Soc. Lond. A 169, 419 (1939)

22. P.O. Löwdin, J. Chem. Phys. 18, 365 (1950)

23. C.C.J. Roothaan, Rev. Mod. Phys. 23, 69 (1951)

24. G.G. Hall, Proc. R. Soc. Lond. A 205, 541 (1951)

25. I. Mayer, Chem. Phys. Lett. 97, 270 (1984)

26. M.S. Gopinathan, K. Jug, Theor. Chim. Acta. 63, 497 (1983)

27. R.F. Nalewajski, J. Mrozek, A. Michalak, Int. J. Quant. Chem. 61, 589 (1997)

28. K. Wiberg, Tetrahedron 1968, 24 (1083)

29. D. Szczepanik, J. Mrozek, J. Math. Chem. (2013). doi:10.1007/s10910-013-0153-8

30. R.F. Nalewajski, J. Math. Chem. 51, 7 (2013)

31. K. Jug, Theor. Chim. Acta. 51, 331 (1979)

32. R.F. Nalewajski, J. Math. Chem. 47, 692 (2010)

33. R.F. Nalewajski, J. Math. Chem. 49, 592 (2011)

34. A.D. Becke, J. Chem. Phys. 98, 5648 (1993)

35. S. Huzinaga, J. Andzelm, M. Klobukowski, E. Radzio-Andzelm, Y. Sakai, H. Tatewaki, Gaussian Basis Sets for Molecular Calculations (Elsevier, Amsterdam, 1984)

36. D.E. Woon, T.H. Dunning Jr, J. Chem. Phys. 98, 1358 (1993)

37. M.W. Schmidt, K.K. Baldridge, J.A. Boatz, S.T. Elbert, M.S. Gordon, J.H. Jensen, S. Koseki, N. Matsunaga, K.A. Nguyen, S. Su, T.L. Windus, M. Dupuis, J.A. Montgomery, J. Comput. Chem. 14, 1347 (1993)

38. U. Varetto, Molekel v5.4.0.8, Swiss National Supercomputing Centre: Manno (Switzerland), (2009)

39. D. Szczepanik, J. Mrozek, Chem. Phys. Lett. 521, 157 (2012) 